\title{
Tissue engineering in urology
}

\author{
Derek J. Matoka, MD; Earl Y. Cheng, MD
}

\begin{abstract}
Tissue engineering encompasses a multidisciplinary approach geared toward the development of biological substitutes designed to restore and maintain normal function in diseased or injured tissues. This article reviews the basic technology that is used to generate implantable tissue-engineered grafts in vitro that will exhibit characteristics in vivo consistent with the physiology and function of the equivalent healthy tissue. We also examine the current trends in tissue engineering designed to tailor scaffold construction, promote angiogenesis and identify an optimal seeded cell source. Finally, we describe several currently applied therapeutic modalities that use a tissue-engineered construct. While notable progress has clearly been demonstrated in this emerging field, these efforts have not yet translated into widespread clinical applicability. With continued development and innovation, there is optimism that the tremendous potential of this field will be realized.
\end{abstract}

Can Urol Assoc J 2009;3(5):403-8

\section{Résumé}

L'ingénierie tissulaire englobe une approche multidisciplinaire axée sur le développement de substituts biologiques en vue de rétablir et de maintenir la fonction normale de tissus lésés. L'article qui suit passe en revue la technologie fondamentale utilisée pour générer des greffons implantables produits par ingénierie in vitro et possédant des caractéristiques in vivo correspondant aux tissus sains équivalents sur les plans physiologique et fonctionnel. Nous examinons également les tendances actuelles en ingénierie tissulaire visant à adapter des échafaudages tissulaires, à promouvoir l'angiogenèse et à dégager une source optimale de cellules implantables. Enfin, nous décrivons plusieurs modalités thérapeutiques actuellement mises en application utilisant un échafaudage créé par ingénierie tissulaire. En dépit de progrès remarquables dans ce domaine en effervescence, les efforts déployés ne se sont pas encore traduits par une applicabilité clinique étendue. Des développements et des percées continus permettent d'être optimiste face au potentiel prodigieux de ce domaine.

\section{Introduction}

Tissue engineering encompasses a multidisciplinary approach geared towards the development of biological substitutes designed to restore and maintain normal function in diseased or injured tissues. Within the field of urology, bioengineers, materials scientists and clinicians have devoted significant resources toward the regeneration of tissues specific to this discipline. While notable progress continues to be evident in the published literature, these efforts have not yet translated into widespread clinical applicability. The exciting potential of this field remains a priority, as the need for donor tissues increases and recent technological advances and the recognition that alternative management modalities are necessary.

\section{Background}

Tissue engineering in urology has evolved in stages, fuelled originally by the search for an alternative reservoir to replace the native bladder. Synthetic, non-biodegradable materials, such as silicone, rubber, polytetrafluoroethylene and polypropylene, were initially used. However, these grafts were quickly encrusted, prone to infection and subject to host-foreign body reactions. ${ }^{1-5}$ The unfavourable outcomes related to these materials led to the development of grafts that were biodegradable, minimally immunogenic and demonstrated the ingrowth of native cells necessary for successful tissue regeneration. Omentum, peritoneum, placenta, pericardium and dura have served as alternatives for the bladder wall. Dura has also been used as a replacement for the tunica albuginea in Peyronie's disease and for urethral suspension in the management of stress urinary incontinence. ${ }^{6-10}$ These initial applications provided a structural substitute but were not conceptually designed to exploit regenerative technology; therefore, the results were inconsistent.

Current advances are focused on the design of implantable tissue-engineered grafts that exhibit characteristics consistent with the physiology and function of the equivalent healthy native tissue. ${ }^{11}$ The success of the graft depends on its ability to provide an appropriate environment for regulating cell behaviour such that adhesion, proliferation, migration and differentiation ultimately result in a graft composed of a population of cells resembling the morphology and phenotype of the desired tissue. 


\section{Concepts}

It is generally accepted that the choice of biomaterial, mechanical properties and its three-dimensional architecture strongly influences a scaffold's ability to promote tissue regeneration. Likewise, a scaffold's design may vary depending on the functional attributes of the tissue being replaced. Essentially, scaffolds are composed of either naturally derived materials, such as collagen or alginate, acellular tissue matrices, or synthetically derived polymers. ${ }^{12}$ Using these biomaterials, 2 strategies are typically used to accomplish the goal of a sustainable graft. The first strategy is "unseeded" technology; it uses either a natural biological or synthetically derived scaffold that encourages the regenerative process by serving as a template for the ingrowth of native cells. A second strategy involves "seeding" the appropriate scaffold with cultured cells prior to implantation into the host. Thus, the regenerative process is not solely dependent on the in vivo growth of cells.

\section{Unseeded technology}

The natural biodegradable acellular extracellular matrix (ECM) has been used as an unseeded substrate for tissue regeneration. The ECM grafts are derived from tissues that have had their cellular components removed through a combination of mechanical and chemical manipulation. However, basic components unique to the extracellular matrix, such as collagen, fibronectin, laminin, glycosaminoglycans (GAGs) and growth factors, are retained, regardless of tissue origin. Thus, the inherent biological activity of the tissue responsible for cell migration, cell-to-cell interaction and cell growth and differentiation is conserved, which is critical to the regenerative process. Within the field of urology, porcine small intestinal submucosa (SIS) and bladder ECM grafts have garnered enthusiastic attention.

Small intestinal mucosa is a thin $(0.1 \mathrm{~mm}$ thick) translucent membrane composed of the submucosal layer of the intestinal wall obtained after extensive mechanical manipulation of a pig's small intestine..$^{13}$ It has been shown to be biologically active due to its unique combination of intrinsic growth factors, cytokines, GAGs and structural proteins. In addition, it does not induce a significant immune response. These characteristics make SIS attractive for tissue regeneration in an unseeded fashion. ${ }^{14,15}$ This result was recognized by Lantz and colleagues, who first used SIS in animal studies as a vascular patch, where it demonstrated tissue-specific regeneration in both arteries and veins. ${ }^{16,17}$
Kropp and colleagues subsequently demonstrated, in both small and large animal models, that SIS alone can promote bladder regeneration when used as an unseeded graft. Histological evaluation demonstrated that the serosal, muscle and mucosal layers of the bladder wall had undergone regeneration. In addition, the resulting tissue was contractile and noted to be functionally innervated based on immunohistochemical staining and urodynamic monitoring. ${ }^{18,19}$ Despite its success, the degree of bladder regeneration can be quite variable and unpredictable. This may be attributed to the variability that exists in the biologic characteristics of the SIS itself or in differences related to manufacturing. Poor regeneration and graft shrinkage were noted in a model of severe bladder disease in which $90 \%$ of a canine bladder was resected and closed prior to subsequent augmentation with SIS. Although histological review demonstrated healthy areas at the periphery of the graft, the authors speculated that the vascularity of a severely damaged bladder may not be able to sustain significant tissue regeneration. ${ }^{20}$ While results have been mixed, SIS has shown that unseeded technology has potential as an application in tissue engineering.

Building on concepts generated through the application of SIS in bladder augmentation, it was speculated that homologous bladder ECM may provide a more suitable substrate for bladder regeneration. ${ }^{21}$ Rat bladder ECM used as a scaffold in an augmentation model appeared nearly identical to the native bladder at 12 weeks after implantation. Additionally, in vivo functionality of hamster, rabbit and dog bladder ECM grafts demonstrated partial detrusor regeneration with improved compliance and functional capacity in augmented rat bladders when compared to a partial cystectomy bladder in controls. ${ }^{22}$ Encouraging results were also observed in a porcine model. Multilayered bladder growth was seen at 1 month and included urothelium, angiogenesis and organized smooth muscle. At longer time intervals, graft shrinkage and a lack of central organized smooth muscle were observed, ${ }^{23}$ likely consistent with inadequate neovascularization. Thus, while unseeded SIS and bladder ECM have shown the ability to participate in the regenerative process, several limitations have prevented this technology from progressing to clinical implementation. Manufacturing that yields predictable biological activity has not consistently been achieved. In addition, there is increased central scarring with contraction in larger grafts, likely due to deficient neovascularization and ischemic changes. With greater understanding of factors that promote ingrowth and neovascularization, the ability to produce a more uniform biodegradable graft may be realized. 


\section{Seeded technology}

A second approach to tissue engineering involves the seeding of cultured cells that are first established in primary culture. Following expansion, cultured cells are seeded onto either a natural or synthetically derived scaffold prior to implantation. This method theoretically does not rely as heavily on the ingrowth of cells from adjacent tissue and is hypothesized to promote faster incorporation of native cells to facilitate tissue regeneration.

Natural and synthetically derived scaffolds have been successfully seeded with bladder urothelial and smooth muscle cells following their harvest, in vitro culture and expansion. In separate works, both bladder ECM and synthetically derived polyglycolic acid (PGA) scaffolds were seeded with autologous urothelial (luminal side) and smooth muscle cells prior to implant in a canine augmentation cystoplasty model. Histologically, the harvested bladders displayed a trilayered structure consisting of urothelial lined lumen, submucosa and smooth muscle. Of significance, functional data demonstrated an increase in bladder capacity and an improvement in overall compliance. Similar results were not achieved for the unseeded scaffolds, which suffered from greater graft contraction. The seeded constructs clearly showed an advantage in promoting the regenerative response over the unseeded group. ${ }^{24,25}$

This work provided the foundation for a landmark clinical trial that used autologous-engineered bladder tissue as an alternative for conventional enterocystoplasty. Atala and colleagues performed augmentation cystoplasty on 7 patients with spina bifida and end-stage bladder disease. PGA composite grafts seeded with autologous urothelial and smooth muscle cells were successfully implanted using an omental wrap to aid in vascularization. ${ }^{26}$ Although histological data at 5-year follow-up displayed a trilayered architecture comprising urothelium, submucosa and muscle, there was significant variability seen in the functional parameters of capacity, compliance and leak point pressure. Only 1 of 7 patients achieved the increase in bladder capacity and improvement in compliance that is routinely observed with conventional enterocystoplasty. In addition, patients were not able to spontaneously void and continued to require intermittent catheterization.

Despite this historic undertaking, it is clear that functional regeneration of normal bladder tissue has yet to be realized. Ultimately, to achieve the potential of a tissue engineered construct, the inherent issues of scaffold design, seeded cell type and promotion of neovascularization must be further explored to expand our understanding of the regenerative process. Progress in these 3 essential areas will lead to improved techniques for successful organogenesis.

\section{Scaffold design}

Scaffold design plays a critical role in the success of an engineered construct and serves as the foundation for directed efforts to achieve neovascularization and implementation of novel seeded cell types. Synthetically derived immunotolerant biomaterials are being meticulously tailored to provide the necessary structural and biological properties required to induce a seeded population of cells to successfully contribute to the dynamic process of tissue regeneration. Poly- $\alpha$-esters, such as poly(L-lactide) (PLLA), PGA and poly(lactide-co-glycolic acid) (PLGA) are commonly used biodegradable synthetic polymers that demonstrate excellent cell and tissue compatibility. These polymers are easily processed into a highly porous, fibrous mesh that is capable of supporting cell ingrowth and proliferation and undergoes degradation through hydrolysis to form standard by-products of respiration.

A significant advantage of using synthetic polymers is the ability to tightly control batch-to-batch composition and maintain excellent reproducibility of the physical properties. ${ }^{27}$ However, these polymers lack the inherent bioactivity that is critical in directing cell activity and fate. While properties of the synthetic scaffold, such as stiffness and surface composition, are important and may indirectly affect cell behaviour, the ability to incorporate signaling machinery and facilitate the delivery of growth factors and cytokines as well as promote angiogenesis could dramatically enhance the regenerative process. Growth factors have been shown to have a marked influence over induction of cell differentiation and phenotype expression. Signaling proteins such as VEGF, PDGF, basic fibroblast growth factor (bFGF), transforming growth factor, and heparin-binding epidermal growth factor (HB-EGF) appear to play a role in the regeneration of tissue within scaffolds and restoration of specialized function, in part through the regulation of vessel density, size and distribution. In particular, bFGF promotes smooth muscle growth and differentiation while also inducing angiogenesis in developing tissues by inducing the proliferation of endothelial cells. Heparin-binding epidermal growth factor is secreted by both epithelial and smooth muscle layers in the bladder and is a mitogenic regulator for both cell types. ${ }^{28,29}$

\section{Neovascularization}

Long-term survival of implanted tissue constructs is dependent on developing adequate vascularization. Therefore, research directed at the promotion of neovascularization is closely tied to scaffold design. Efforts have been directed toward incorporation of the growth factor into a scaffold with subsequent controlled local delivery to enhance the 
regenerative response and promote angiogenesis. When both bFGF and VEGF are integrated into a synthetically derived scaffold, local angiogenesis has been observed. ${ }^{30}$ These scaffolds have been supplemented with various factors, typically using a process of simple adsorption to initially "load" the scaffold. However, this bolus delivery of factors can have unpredictable systemic effects. In addition, uncontrolled release may lead to premature degradation or inactivation. Thus, a protected and controlled release is essential for the delivery of relevant mediators or growth factors. Research efforts directed at tailoring the synthesis and composition of scaffolds for this purpose have been promising. Through application of a thermally induced phase separation technique that yielded a highly porous construct, a biodegradable, elastomeric poly(ester urethane)urea (PEUU) scaffold was created that demonstrated controlled release of bFGF. ${ }^{31}$ The designed porosity as well as the incorporation of heparin into the scaffold contributed to the favourable release pattern of bFGF. Other work has also demonstrated the influence of heparin on the controlled release of bFGF when synthetically integrated into a scaffold. Heparin immobilized PLGA scaffolds loaded with bFGF showed a notable in vivo angiogenic response, while release assays displayed an initial burst followed by a sustained release over a 3-week period. ${ }^{32}$ Finally, nanotechnology or the field of molecular self-assembly is poised to contribute to regenerative medicine in a similar fashion. Unlike many conventional polymeric molecules, self-assembling molecules can be injected as a solution into a site and then triggered to assemble through alterations in $\mathrm{pH}$, ionic charge or aggregation of biomacromolecules. Cells may be embedded within a self-assembling molecule, providing a means to immobilize the cell at a specific site. A similar concept may be used for the design of scaffolds capable of loading cells and the sustained release of a number of factors that could benefit the regenerative process.

\section{Seeded cell type: the application of stem cells}

Despite the role of autologous cell therapy in advancing tissue engineering, concerns regarding the functional characteristics of cells harvested from a dysfunctional or neurogenic source have been raised. ${ }^{33-35}$ In vitro work has suggested that conversion to a normal phenotype from a diseased source may not occur. In addition, harvesting these cells can be difficult and potentially morbid, their proliferative capacity may be decreased and functional quality reduced from in vitro culturing. Therefore, emphasis has also been placed on exploring the potential of both pluripotent and multipotent autologously derived stem cells that are capable of self-renewal and tissue-specific differentiation that will not illicit an unwanted immune response. ${ }^{34}$
Stem cells are, by definition, undifferentiated and characterized by their ability to undergo both self-renewal and differentiation. The mature progeny that are produced consist of both non-renewing progenitors and terminally differentiated effector cells. ${ }^{34,36}$ Stem cells are classified according to their potency, where the hierarchic ordering ranges from totipotency (zygote and its offspring cells of the morula) to pluripotency (embryonic stem cells and embryonic germ cells) and multipotency (haematopoietic and mesenchymal stem cells). Unipotent cells differentiate into only one cell type and exhibit little or no capacity for self-renewal. ${ }^{34}$

The potential use of stem cells in all disciplines of regenerative medicine has been heavily investigated. In particular, bone marrow is a vast resource for tissue-derived adult stem cells. Bone marrow-derived stromal cells have shown the ability to differentiate into bone, fat, cartilage, muscle and other lineages, as well as non-mesenchymal tissue cell types such as endodermal or neural cells. Mesenchymal stem cells (MSCs) are highly proliferative, undergo a relatively wide range of differentiation and can be routinely isolated, making them an attractive source for multiple applications. ${ }^{34}$ Mesenchymal stem cells have been used in the augmentation of a rat bladder. Small intestinal submucosa was seeded with MSCs derived from rat bone marrow prior to augmenting the rat bladder after partial cystectomy. A trilayered cellular morphology was noted at 3 months. Both smooth muscle and urothelial cells were observed. A similar architecture was also apparent in the control group that was not seeded with MSCs, although a longer duration was required to distinguish the three layers. ${ }^{37}$ Although migration of cells from native tissue more than likely contributed to the findings, the transplanted stem cells imparted an observable advantage. However, the responsible mechanism remains to be elucidated. Clearly, the use of biocompatible scaffolds in combination with autologous stem cells for regenerative purposes in urology is gaining momentum, with future investigative work benefiting from the strong foundation already in place.

\section{Clinical application of tissue engineering}

Tissue engineering has made strides towards clinical implementation for the management of urological disease other than bladder. We examine several applied therapeutic modalities using the concepts described above.

\section{Urethra}

Urethral reconstructive surgery continues to evolve, using new techniques and a variety of tissues. Success and choice of technique generally are dependent on the availability of 
well-vascularized autologous donor tissue. When viable tissue is not available, tissue engineering has the potential to provide needed tissue substitutes. ${ }^{38}$ Small intestinal submucosa has been used in the management of anterior urethral stricture disease with results comparable to buccal mucosa grafts. In one study, 50 patients were managed with an SIS graft placed in an onlay fashion. At a mean follow-up of 31 months, the success rate was $80 \% .{ }^{39} \mathrm{~A}$ second study reported $85 \%$ success when SIS was used as either an inlay or onlay patch graft with a mean follow-up of 21 months. However, when used ventrally, the outcome was not as favourable, with a failure reported in 6 of 10 adult patients. ${ }^{40}$ Acellular bladder matrix has also been used in the management of complex urethral strictures. The graft was placed in an onlay fashion to the urethral plate in 28 patients. At a mean follow-up of 37 months, a successful outcome was observed in 24 patients. ${ }^{41}$

\section{Corporal body}

Advances in tissue engineering have provided reconstructive alternatives for the management of penile structural disorders. Small intestinal submucosa has demonstrated safe and reliable use in a paediatric population requiring corporal body grafting. ${ }^{42}$ At a mean follow-up of 20 months, there was no evidence of graft shrinkage or penile deformation and a straight phallus was noted with either spontaneous or induced erection. Subsequent work has demonstrated that 1-ply SIS has superior results to 4-ply SIS in this setting. ${ }^{43}$ Small intestinal submucosa grafts have also been used in the management of Peyronie's disease following plaque incision in adults. Small intestinal submucosa was used to successfully manage symptomatic penile curvature in 148 of $162(91 \%)$ patients with a mean follow-up of 38 months. The authors describe no associated comorbidities or adverse reactions related to the SIS and report a potency rate of $79 \%{ }^{44}$

\section{Conclusion}

Clearly, there has been significant progress in the field of tissue engineering. However, despite the advances in technique and methodology, and a continued high level of interest, widespread clinical applicability remains elusive. Understanding how cells participate in the regenerative process as well as the mechanisms that influence their involvement are crucial to identifying the key cellular and molecular participants in organogenesis. By achieving these goals, researchers will be able to better tailor their advances in materials and biology to match the needs of each system. While continued development and additional innovation are necessary before potential broad applications are realized, there is cautious optimism that tissue engineering will play an increasing role in the management of a spectrum of urological disease.

From the Department of Pediatric Urology, Children's Memorial Hospital and the Feinberg School of Medicine, Northwestern University, Chicago, IL

This paper has been peer-reviewed.

Competing interests: None declared.

\section{References}

1. Kurdish HG. The use of polyvinyl sponge for experimental cystoplasty. J Urol 1957;78:232.

2. Swinney JT, Walden DN. Urinary tract substitution. Br J Urol 1961;33:414-27.

3. Agishi T, Nakazono M, Kiraly RJ, et al. Biodegradable material for bladder reconstruction. J Biomed Mater Res 1975;9:119-31.

4. Bohne AW, Osborn RW, Hettle PJ. Regeneration of the urinary bladder in the dog, following total cystectomy. Surg Gynecol Obstet 1955;100:259-64.

5. Scott R, Mohammed R, Gorham SD, et al. The evolution of a biodegradable membrane for use in urological surgery. A summary of 109 in vivo experiments. Br J Urol 1988;62:26-31.

6. Fishman IJ, Flores FN, Scott FB, et al. Use of fresh placental membranes for bladder reconstruction. J Urol 1987;138:1291-4.

7. Gorham S, McCafferty I, Baraza R, et al. Preliminary development of a collagen membrane for use in urological surgery. Urol Res 1984;12:295-9.

8. Kambic H, Kay R, Chen JF, et al. Biodegradable pericardial implants for bladder augmentation: a 2.5-year study in dogs. J Urol 1992; 148:539-43.

9. Kropp BP, Eppley BL, Prevel CD, et al. Experimental assessment of small intestinal submucosa as a bladder wall substitute. Urology 1995;46:396-400.

10. Kelami A. [The use of human dura in urology, except as a substitute of urinary bladder (author's transl)]. Urol Int 1976;31:290-6.

11. Wood D, Southgate J. Current status of tissue engineering in urology. Curr Opin Urol 2008;18:564-9.

12. Atala A. Technology insight: applications of tissue engineering and biological substitutes in urology. Nat Clin Pract Urol 2005;2:143-9.

13. Badylak SF, Lantz GC, Coffey A, et al. Small intestinal submucosa as a large diameter vascular graft in the dog. I Surg Res 1989;47:74-80.

14. Badylak SF. Xenogeneic extracellular matrix as a scaffold for tissue reconstruction. Transpl Immunol 2004; 12:367-77.

15. Voytik-Harbin SL, Brightman A0, Kraine MR, et al. Identification of extractable growth factors from small intestinal submucosa. J Cell Biochem 1997;67:478-91.

16. Lantz GC, Badylak SF, Coffey AC, et al. Small intestinal submucosa as a small-diameter arterial graft in the dog. I Invest Surg 1990;3:217-27.

17. Lantz GC, Badylak SF, Coffey AC, et al. Small intestinal submucosa as a superior vena cava graft in the dog. J Surg Res 1992;53:175-81.

18. Kropp BP, Rippy MK, Badylak SF, et al. Regenerative urinary bladder augmentation using small intestinal submucosa: urodynamic and histopathologic assessment in long-term canine bladder augmentations. J Urol 1996;155:2098-104.

19. Kropp BP, Sawyer BD, Shannon HE, et al. Characterization of small intestinal submucosa regenerated canine detrusor: assessment of reinnervation, in vitro compliance and contractility. J Urol 1996;156: 599-607.

20. Zhang Y, Frimberger D, Cheng EY, et al. Challenges in a larger bladder replacement with cell-seeded and unseeded small intestinal submucosa grafts in a subtotal cystectomy model. BJU Int 2006;98:1100-5.

21. Probst $M$, Dahiya R, Carrier $S$, et al. Reproduction of functional smooth muscle tissue and partial bladder replacement. Br J Urol 1997;79:505-15.

22. Piechota $H J$, Dahms SE, Probst $M$, et al. Functional rat bladder regeneration through xenotransplantation of the bladder acellular matrix graft. Br J Urol 1998;81:548-59. 
23. Brown AL, Farhat W, Merguerian PA, et al. 22 week assessment of bladder acellular matrix as a bladder augmentation material in a porcine model. Biomaterials 2002;23:2179-90.

24. Yoo JJ, Meng J, Oberpenning F, et al. Bladder augmentation using allogenic bladder submucosa seeded with cells. Urology 1998;51:221-5.

25. Oberpenning F, Meng J, Yoo JJ, et al. De novo reconstitution of a functional mammalian urinary bladder by tissue engineering. Nat Biotechnol 1999:17:149-55.

26. Atala A, Baver SB, Soker S, et al. Tissue-engineered autologous bladders for patients needing cystoplasty. Lancet 2006;367:1241-6.

27. Harrington DA, Sharma AK, Erickson BA, et al. Bladder tissue engineering through nanotechnology. World J Urol 2008;26:315-22

28. Freeman MR, Yoo JJ, Raab G, et al. Heparin-binding EGF-like growth factor is an autocrine growth factor for human urothelial cells and is synthesized by epithelial and smooth muscle cells in the human bladder. J Clin Invest 1997;99:1028-36.

29. Park JM, Adam RM, Peters CA, et al. AP-1 mediates stretch-induced expression of HB-EGF in bladder smooth muscle cells. Am J Physiol 1999;277:C294-301.

30. Perets $A$, Baruch $Y$, Weisbuch $F$, et al. Enhancing the vascularization of three-dimensional porous alginate scaffolds by incorporating controlled release basic fibroblast growth factor microspheres. J Biomed Mater Res A 2003;65:489-97.

31. Guan J, Stankus JJ, Wagner WR. Biodegradable elastomeric scaffolds with basic fibroblast growth factor release. J Control Release 2007;120:70-8.

32. Chung HJ, Kim HK, Yoon JJ, et al. Heparin immobilized porous PLGA microspheres for angiogenic growth factor delivery. Pharm Res 2006;23:1835-41.

33. Lewis JM, Cheng EY. Non-traditional management of the neurogenic bladder: tissue engineering and neuromodulation. ScientificWorldJournal 2007;7:1230-41.

34. Becker C, Jakse G. Stem cells for regeneration of urological structures. Eur Urol 2007;51:1217-28.

35. Beqai SH, Donovan JL, Liu DB, et al. Role of basic fibroblast growth factor in the neuropathic bladder phenotype. J Urol 2005; 174:1699-703.
36. Blau HM, Brazelton TR, Weimann JM. The evolving concept of a stem cell: entity or function? Cell 2001;105:829-41.

37. Chung SY, Krivorov NP, Rausei V, et al. Bladder reconstitution with bone marrow derived stem cells seeded on small intestinal submucosa improves morphological and molecular composition. J Urol 2005; 174:353-9.

38. Yamzon J, Perin L, Koh CJ. Current status of tissue engineering in pediatric urology. Curr Opin Urol 2008; 18:404-7

39. Fiala R, Vidlar A, Vrtal R, et al. Porcine small intestinal submucosa graft for repair of anterior urethral strictures. Eur Urol 2007;51:1702-8; discussion 1708.

40. Albers $P$. Tissue engineering and reconstructive surgery in urology. Eur Urol 2007;52:1579.

41. El-Kassaby AW, Retik AB, Yoo JJ, et al. Urethral stricture repair with an off-the-shelf collagen matrix. J Urol 2003; 169:170-3; discussion 173.

42. Kropp BP, Cheng EY, Pope JCT, et al. Use of small intestinal submucosa for corporal body grafting in cases of severe penile curvature. J Urol 2002;168:1742-5; discussion 1745.

43. Leslie JA, Cain MP, Kaefer $M$, et al. Corporeal grafting for severe hypospadias: a single institution experience with 3 techniques. J Urol 2008; 180(Supp|4):1749-52; discussion 1752.

44. Knoll LD. Use of small intestinal submucosa graft for the surgical management of Peyronie's disease. J Urol 2007; 178:2474-8; discussion 2478.

Correspondence: Dr. Earl Y. Cheng, Department of Pediatric Urology, Children's Memorial Hospital and the Feinberg School of Medicine, Northwestern University, 2300 Children's Plaza, Box 24, Chicago, IL 60614; fax: 773-880-3339; echeng@childrensmemorial.org 